\title{
Trapping parameters comparison between cable sections from different service conditions by a new trapping-detrapping model
}

\author{
Ning Liu and George Chen \\ University of Southampton \\ University Road \\ Southampton, SO17 1BJ, UK
}

\author{
Yang $\mathrm{Xu}$ \\ Xi'an Jiaotong University \\ Xi'an, Shaanxi, 710049, China
}

\begin{abstract}
Space charge formation will cause electric field enhancement at certain location in the polymeric materials, especially under HVDC insulation condition. In our previous works, a model has been developed which is suitable for analysing the space charge data from depolarization test to calculate the material trapping parameters. In the present paper, two cross-linked polyethylene (XLPE) cables, taken from HVAC service condition of $220 \mathrm{kV}$ for 12 years and for 8 years, were peeled as $100-200 \mu \mathrm{m}$ films from the outer to inner layer of insulation. Through the comparison between trapping parameters estimated from our model, it has been found that samples from inner insulation layers for both cables have the deepest trap depth and the greatest deep trap density, indicating samples from inner layers have the severest ageing.
\end{abstract}

\section{INTRODUCTION}

In high voltage insulation systems, particularly under high voltage direct current (HVDC) condition, space charges tend to accumulate in polymeric materials through charge injection at electrode interface and ionization process within bulk insulation. The presence of space charge will modify electric field in the area adjacent to electrodes or within the bulk sample. As a consequence, it will give rise to degradation or ageing in polymeric materials and potentially lead to early failure of materials.

Space charge accumulation could be thought as a consequence of charge capturing by localized states at certain energy level, namely 'traps'. In the recent decade, many efforts have been put on the investigation of trapping parameters of insulation materials. In [1], a simple trapping-detrapping model based on two energy levels was derived from the Schottky injection mechanism. The model considers the whole traps in materials as two types: shallow traps and deep traps. These traps might be correlated with physical and chemical defects in materials. The physical defects could be recognized as changes of morphological structure, crystallinity and molecular weights whereas chemical defects refer to oxidized sites and other new chemical constituents [2, 3]. Higher degree of ageing should cause changes in parameters of traps in materials, typically trap density, trap depth and cross-sectional trapping area. Thereafter in [4], based on the model in [1], trapping parameters of low-density polyethylene (LDPE) were estimated through a curve fitting with depolarization data. Also in [5], by using the same approach, we compared trapping parameters estimated from the gamma-irradiated
LDPE and normal LDPE materials. It was found that trapping parameters, especially for deep traps, experienced obvious changes after irradiation.

In the present paper, we improved the simple model in [1] by taking account of charge retrapping process in materials. XLPE cables aged for different years were peeled by microtoming into 100 200 $\mu \mathrm{m}$ films and these films were grouped in accordance with operation years and radical distance to core conductor. And pulsed electro-acoustic (PEA) depolarization measurement was applied on these samples to detect the charge profile dynamics inside materials. The improved model could be used to analyze the space charge decay and allows quantitative estimation of trapping parameters of those different samples.

\section{MODEl OF CHARGE DYNAMICS}

Poole-Frenkel effect occurs in the material of wide band-gap (e.g. insulating material or insulator) where electrons/holes can reside in. The effect demonstrates the Pool-Frenkel mechanism that the potential barrier height of columbic attractive traps is deducted by columbic force between these charge trapping centres and charge carriers. By considering the effect of poling electric field in equation (1), the trap depth $E_{t}$ could be reduced in the electric field direction by value $\Delta V_{F}$, i.e.:

$$
E_{t}^{\prime}=E_{t}-\Delta V_{F}(E)
$$

where the maximum reduced height $\Delta V_{F m}(E)$ of the barrier in the electric field direction is

$$
\Delta V_{F m}(E)=-2\left(\frac{e^{3} E}{4 \pi \varepsilon_{0} \varepsilon_{r}}\right)^{\frac{1}{2}}
$$

This maximum reduced height happens when Columbic force between electrons/holes and ionised donors/acceptors (i.e. traps) equals to electrostatic force under the applied field $E$ (further details in [6]). Thus, the rate of thermal excitation of trapped electrons/holes from localized states to the conduction/valence band could be given by:

$$
R_{e s c}=n_{t} v_{0} \exp \left(-\frac{E_{t}^{\prime}}{k T}\right)
$$

where $n_{t}$ is the trapped charge density, $T$ is temperature ( $300 \mathrm{~K}$ used in the present paper), $k$ is the Boltzmann constant $\left(\sim 1.38 \times 10^{-23} \mathrm{~m}^{2} \mathrm{~s}^{-2} \mathrm{~K}^{-1} \mathrm{~kg}\right)$, and $v_{0}$ is the attempt to escape frequency $\left(\sim 2 \times 10^{13} \mathrm{~Hz}\right.$ at room temperature [7]). 
However, our model is applied to the condition when the applied electric field is removed. For the sake of simplicity, we neglect the Pool-Frenkel effect caused by local space charge field, hence $E_{t}^{\prime}$ could be approximated as $E_{t}$.

Moreover, the rate of capturing by trap sites should be proportional to the number density of free charges which have been released into the conduction/valence band $n_{f}$ and the number of unoccupied trap sites density $\left(N-n_{t}\right)$, and $N$ is total trap density.

$$
R_{\text {cap }}=n_{f}\left(N-n_{t}\right) v_{t h} S
$$

where $v_{t h}$ is the thermal velocity $\left(=3.7 \times 10^{5} \mathrm{~m} / \mathrm{s}\right.$ for holes in polyethylene material [4]) of electrons or holes and $S$ is capture cross section area of trap sites for charge carriers.

In [1] and [7], these models consider observed charges as trapped charges. Actually, the free charge carriers, or said mobile charges will flow away from local space charge region by extracting from adjacent electrode or flowing into the other side of electrode dependent upon the direction of coulombic force it experienced. However, in models applied in [1] and [7], those mobile charges were thought flowing very instantly from original location. Here in our model, we take consideration of transit time $\Delta t$ for detrapped charges' outflowing from local charge region. And we made an important assumption that during $\Delta t$, the charges might be recaptured by unoccupied traps and the free charge carrier density generated by excited charges could be expressed as: $n_{f}=\int_{t}^{t+\Delta t} R_{e s c} d t$. For the estimation of $\Delta t$, we use: $\Delta t=\frac{d}{v_{d}}$. Here, $d$ is the averaged distance from original location to the boundary of space charge region. Typically, in our measurements, the positive charge peak is dominant. Since the width of positive space charge region is around $80 \mu \mathrm{m}$, therefore, we approximated $d$ as half width of the positive peak, i.e. $40 \mu \mathrm{m}$. And under electric field at an order of $10^{6} \mathrm{~V} / \mathrm{m}$, which is the approximated electric field after removal of the external voltage in our volts-off measurements, the drift velocity is at an order of $10^{5} \mathrm{~m} / \mathrm{s}$ [5]. As a result, the transit time $\Delta t$ could be approximated as: $\Delta t=\frac{d}{v_{d}}=4 \times$ $10^{-10} \mathrm{~s}$. As this time is too short for charge escaping rate to have a substantial variation, we could estimate free charge carrier density as: $n_{f}=R_{e s c} \Delta t$. And changing rate of trapped charge density $n_{t}$ is:

$\frac{d n_{t}}{d t}=R_{\text {cap }}-R_{\text {esc }}=n_{t} v_{0} \exp \left(-\frac{E_{t}}{k T}\right)\left[\Delta t\left(N-n_{t}\right) v_{t h} S-1\right](5)$ Solving equation (5), we could have solution in form of:

$$
n_{t}=a \operatorname{coth}(b t+c)-a
$$

where $a$ and $b$ were shortened for:

$$
\begin{gathered}
a=\frac{1-\Delta t\left(N-n_{t}\right) v_{t h} S}{2 \Delta t v_{t h} S} \\
b=v_{0} \exp \left(-\frac{E_{t}}{k T}\right) \frac{\left[1-\Delta t\left(N-n_{t}\right) v_{t h} S\right]}{2}
\end{gathered}
$$

And for the initial condition, trapped charge density $\mathrm{n}_{0}$ at $t=0$, we have:

$$
n_{0}=n_{t}(0)=\operatorname{acoth}(c)-a
$$

Furthermore, there is evidence [8] stating the point that in dielectrics deeper traps should have a smaller cross section area. Physically, it can be explained that smaller capture radius will give rise to a greater coulombic attractive force upon on charge carrier, hence forming a deeper trap, which is harder for charge carrier to escape. Especially in [9], it was proposed that he binding energy $W$ of a coulombic trap to charge carrier is inversely proportional to radius of the trap $r$. The binding energy $W$ directly determines the trap depth $E_{t}$. The larger $W$ becomes, the tighter the charge carrier bounds to the trap, i.e. trap depth will be deeper. Here, we assumed that $E_{t}$ is proportional to $W$. Since trap cross-sectional area $S=\pi r^{2}$, we could have $S$ inversely proportional to $E_{t}^{2}$.

Here, according to references cross sectional area values in some previous works on trapping parameters estimation [4][5][10], the reasonable value of cross section should be at order from $10^{-16}$ to $10^{-18}$. Furthermore in [11], it was proposed that cross section area should be field-dependent as well. In the present paper, in light of these studies on trapping cross section area, we could approximate the value for cross section area of traps at field $\mathrm{E}=10^{6} \mathrm{~V} / \mathrm{m}$ that at depth $E_{t 1}=$ $1 \mathrm{eV}, \mathrm{S}_{1}=1 \times 10^{-16} \mathrm{~m}^{2}$. Therefore for any trap at depth $E_{t}$, the capture cross section can be expressed as:

$$
S=\left(\frac{E_{t 1}}{E_{t}}\right)^{2} S_{1}
$$

Combining equations (7), (8), (9) and (10), we could obtain values of trapping parameters $E_{t}, N, S$ and intial trapped charge $n_{0}$ by knowing fitted values $a, b$ and $c$.

\section{SAMPLE PREPARATION}

Cross-linked polyethylene (XLPE) cable sections were from high voltage cables which had been used for 12 years and 8 years operated at AC $220 \mathrm{kV}$. The cable structure and the size are illustrated in Figure 1. The XLPE of cable insulator was sliced to film by a rotary skiver (a cutting machine to make film by rotation) from the surface of cable insulator. The thickness of obtained samples was 100-200 $\mu \mathrm{m}$ with smooth surface. For the removal of volatile chemicals in the film, the cut films were treated in vacuum oven at $80^{\circ} \mathrm{C}$ for 48 hours for degassing.

The film samples for the experiment were classified to 6 parts according to the distance from the surface of cable insulator as seen in Figure 1. In this paper, three different positions were selected as the outer $(0 \sim 5 \mathrm{~mm}$ from surface), middle (14 18 $\mathrm{mm})$, and inner (23 30 $\mathrm{mm}$ ) layer for 12-year-operated cable and the outer $(0 \sim 5 \mathrm{~mm}$ from surface), middle $(10 \sim 15 \mathrm{~mm})$, and inner (20 27 mm) layer for 8-year-operated cable.

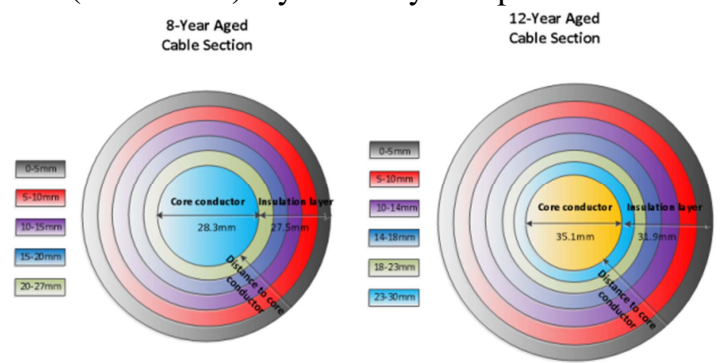

Figure 1. Cable cross section and XLPE insulator positions for charge dynamics measurement.

\section{EXPERIMENTAL PROCEDURES}


The pulsed electroacoustic (PEA) technique was used for observing dynamics of charge profiles and measurements were made for 30 minutes after the removal of the applied voltage. For XLPE films with different thickness, the applied voltage was adjusted so the applied field was fixed at $40 \mathrm{kV} / \mathrm{mm}$ for all the samples. The time of the applied voltage was 6 minutes.

\section{Results OF CHARGE DYNAMICS}

Typical results of charge decay dynamics of 12-yearoperated and 8-year-operated XLPE films were shown in Figures 2 to 7 . Particularly for the results in figure 5, only injected positive charge peak was observed. And for figures 2 and 6, bipolar injected charges were observed but not obvious and the positive image charge peak on the cathode was not displayed on scope. But for figures 3, 4 and 7, clear bipolar injected charges decay could be observed. These might be caused by two reasons: (i) limited resolution of the PEA system, (ii) inner layer contains more deep traps, which can capture electrons more easily.

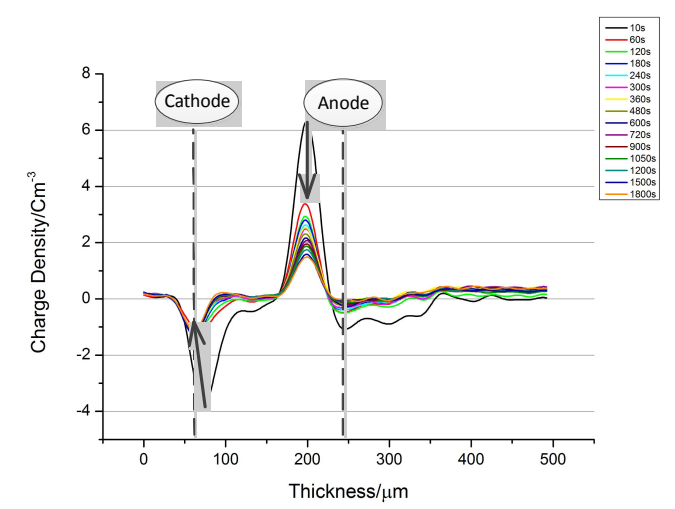

Figure 2: Charge profiles for $160 \mu \mathbf{m}$ thick 12-year-operated XLPE at outerlayer after the removal of $6.4 \mathrm{kV}$ charge.

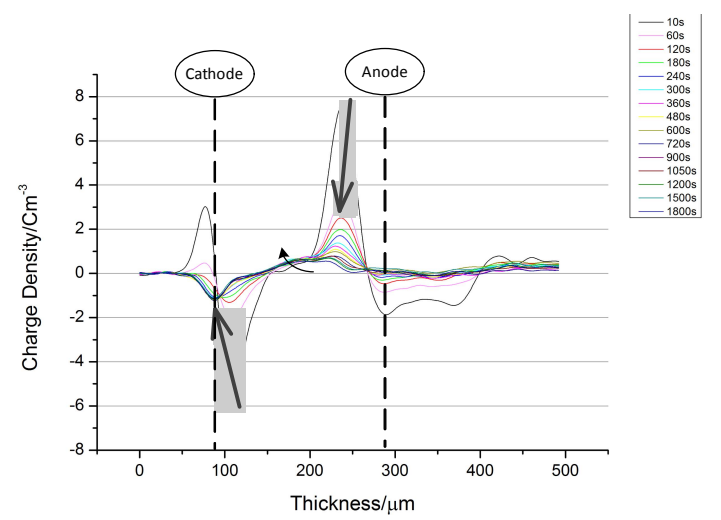

Figure 3: Charge profiles for 180 $\mathrm{mm}$ thick 12-year-operated XLPE at middlelayer after the removal of $7.2 \mathrm{kV}$ charge.

\section{ESTIMATION OF TRAPPING PARAMETERS}

To calculate the total trapped charge $Q$ amount inside the bulk, equation (11) applies.

$$
Q=\int_{0}^{l}|\rho(x, t)| S d x
$$

where $l$ is the thickness of positive charge layer, $S$ is the electrode area, $t$ is the depolarization time, and $x$ is the coordinate on horizontal axis. Therefore, the density of the trapped charges $n_{t}$ is:

$$
n_{t}=\frac{Q}{l \cdot S \cdot q}
$$

PEA measurement is rather sensitive to environmental factors such as temperature, moisture, mechanical stresses and silicone oil applied for acoustic coupling. Therefore, even we applied the same electric field with an identical stressing time, measurement results may more or less differ from each other. To minimize the occasional errors caused by those uncontrollable factors, we selected some similar results from measurements of each layer and average trapped charge.

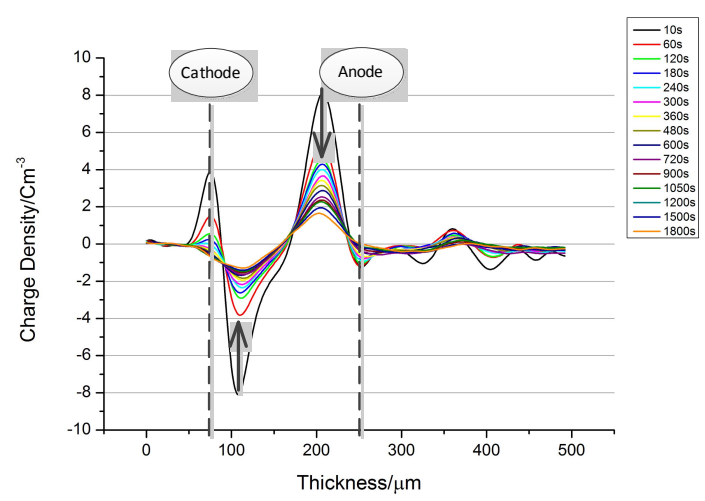

Figure 4: Charge profiles for $150 \mu \mathrm{m}$ thick 12 -year-operated XLPE at innerlayer after the removal of $6 \mathrm{kV}$ charge.

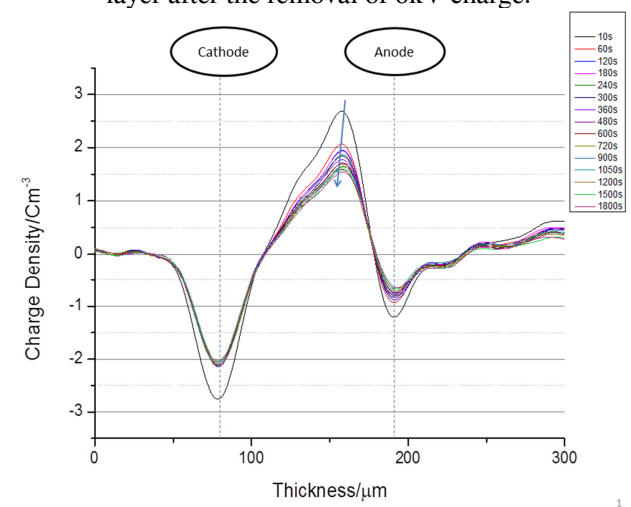

Figure 5: Charge profiles for $110 \mu \mathrm{m}$ thick 8-year-operated XLPE at out-layer after the removal of $4.5 \mathrm{kV}$ charge.

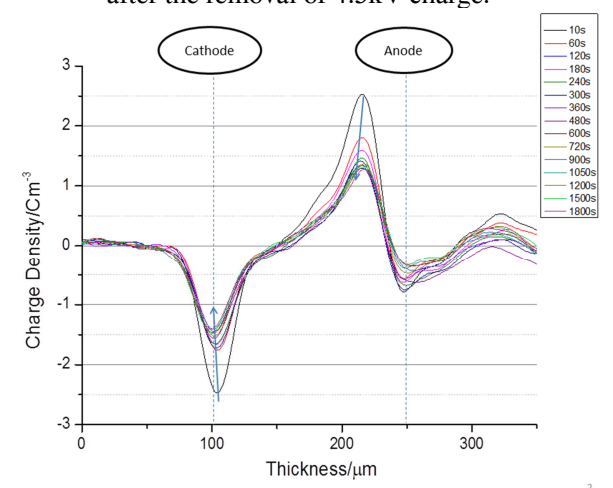

Figure 6: Charge profiles for $150 \mu \mathrm{m}$ thick 8-year-operated XLPE at innerlayer after the removal of $6 \mathrm{kV}$ charge. 


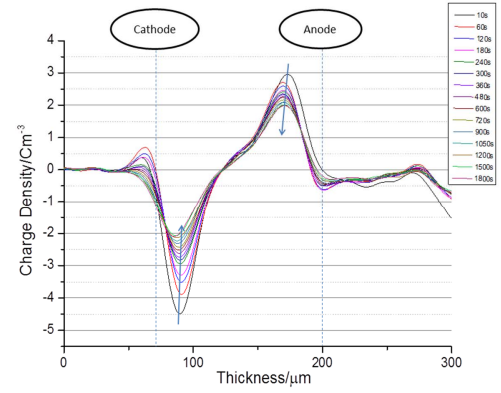

Figure 7: Charge profiles for $115 \mu \mathrm{m}$ thick 8-year-operated XLPE at innerlayer after the removal of $4.6 \mathrm{kV}$ charge.

When we extend the model described by equation (13) to two equivalent level traps:

$$
n_{t}=a \operatorname{coth}(b t+c)-a+d \operatorname{coth}(e t+f)-d \text { (13) }
$$

The plots of decay experimental data scattering fitted with equation (13) were shown in Figure 8.
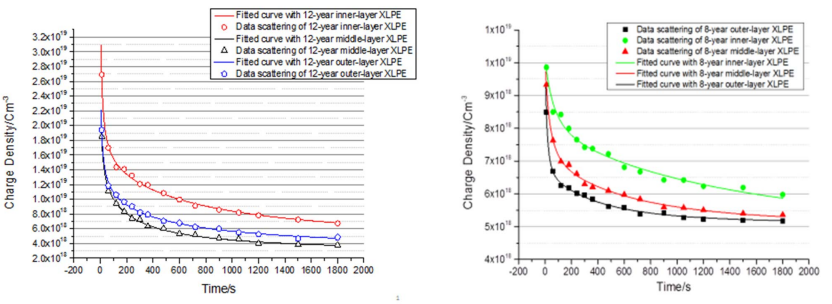

Figure 8: Curve fitting results of XLPE peelings from three layers using equation (13) respectively for 12-year cable (left) and 8-year cable (right).

And the trapping parameters of both types of samples could be obtained as shown in Table 1.

TABLE I

Trapping Parameters Of Traps With Shallow And DeEp Equivalent ENERGY LeVELS From EQUATION (13)

\begin{tabular}{|c|c|c|}
\hline \multirow{2}{*}{ Trapping parameters } & \multicolumn{2}{|c|}{ 12-year XLPE samples from inner layer } \\
\hline & Shallow traps & Deep traps \\
\hline $\mathrm{N} / \mathrm{m}^{-3}$ & $4.95 \times 10^{19}$ & $5.91 \times 10^{19}$ \\
\hline $\mathrm{E}_{\mathrm{t}} / \mathrm{eV}$ & 0.834 & 0.921 \\
\hline $\mathrm{S} / \mathrm{m}^{2}$ & $1.44 \times 10^{-16}$ & $1.18 \times 10^{-16}$ \\
\hline \multirow{2}{*}{ Trapping parameters } & \multicolumn{2}{|c|}{ 12-year XLPE samples from middle layer } \\
\hline & Shallow traps & Deep traps \\
\hline \multirow{3}{*}{$\begin{array}{l}\mathrm{N} / \mathrm{m}^{-3} \\
\mathrm{E}_{\mathrm{t}} / \mathrm{eV} \\
\mathrm{S} / \mathrm{m}^{2}\end{array}$} & \multirow{3}{*}{$\begin{array}{c}4.78 \times 10^{19} \\
0.826 \\
1.47 \times 10^{-16}\end{array}$} & $5.53 \times 10^{19}$ \\
\hline & & 0.895 \\
\hline & & $1.25 \times 10^{-16}$ \\
\hline \multirow{2}{*}{ Trapping parameters } & \multicolumn{2}{|c|}{ 12-year XLPE samples from outer layer } \\
\hline & Shallow traps & Deep traps \\
\hline $\mathrm{N} / \mathrm{m}^{-3}$ & \multirow{3}{*}{$\begin{array}{c}4.80 \times 10^{19} \\
0.828 \\
1.46 \times 10^{-16}\end{array}$} & $5.73 \times 10^{18}$ \\
\hline $\mathrm{E}_{\mathrm{t}} / \mathrm{eV}$ & & 0.905 \\
\hline $\mathrm{S} / \mathrm{m}^{2}$ & & $1.22 \times 10^{-16}$ \\
\hline \multirow{2}{*}{ Trapping parameters } & \multicolumn{2}{|c|}{ 8-year XLPE samples from inner layer } \\
\hline & Shallow traps & Deep traps \\
\hline \multirow{3}{*}{$\begin{array}{l}\mathrm{N} / \mathrm{m}^{-3} \\
\mathrm{E}_{\mathrm{t}} / \mathrm{eV} \\
\mathrm{S} / \mathrm{m}^{2}\end{array}$} & \multirow{3}{*}{$\begin{array}{c}4.95 \times 10^{19} \\
0.835 \\
1.43 \times 10^{-16} \\
\end{array}$} & $5.92 \times 10^{19}$ \\
\hline & & 0.918 \\
\hline & & $1.19 \times 10^{-16}$ \\
\hline \multirow{2}{*}{ Trapping parameters } & \multicolumn{2}{|c|}{ 8-year XLPE samples from middle layer } \\
\hline & Shallow traps & Deep traps \\
\hline \multirow{3}{*}{$\begin{array}{l}\mathrm{N} / \mathrm{m}^{-3} \\
\mathrm{E}_{\mathrm{t}} / \mathrm{eV} \\
\mathrm{S} / \mathrm{m}^{2}\end{array}$} & \multirow{3}{*}{$\begin{array}{c}4.81 \times 10^{19} \\
0.8217 \\
1.48 \times 10^{-16} \\
\end{array}$} & $5.64 \times 10^{19}$ \\
\hline & & 0.8915 \\
\hline & & $1.26 \times 10^{-16}$ \\
\hline \multirow{2}{*}{ Trapping parameters } & \multicolumn{2}{|c|}{ 8-year XLPE samples from outer layer } \\
\hline & Shallow traps & Deep traps \\
\hline
\end{tabular}

\begin{tabular}{c|c|c}
\hline $\mathrm{N} / \mathrm{m}^{-3}$ & $4.68 \times 10^{19}$ & $5.43 \times 10^{19}$ \\
$\mathrm{E}_{\mathrm{t}} / \mathrm{eV}$ & 0.806 & 0.877 \\
$\mathrm{~S} / \mathrm{m}^{2}$ & $1.54 \times 10^{-16}$ & $1.30 \times 10^{-16}$ \\
\hline
\end{tabular}

\section{CONCLUSION}

From the Table I, we could draw the conclusions as:

1. For both different-year aged cable sections, trap density and trap depth of deep traps are generally larger than that from the other two layers. This should be caused by the severest condition (highest temperature and electric field) experienced during operation duration.

2. Look into details of 12-year-operated cable, the trapped parameters of outer-layer XLPE is greater than that of middle layer. This is probably due to that even middle-layer in cable experienced a more serious service condition, but outer region had more sufficient contact with oxygen during operation, which might induce more oxidized product into such layer.

3. The model present in this paper can provide a tool to monitor ageing or degradation in materials.

\section{REFERENCES}

[1] G. Chen and Z. Xu, "Charge trapping and detrapping in polymeric materials," Journal of Applied Physics, vol. 106, no. 12, p. 123707, 2009.

[2] D. Marsacq, P. Hourquebie, L. Olmedo, and H. Janah, "Effects of physical and chemical defects of polyethylene on space charge behaviour," in Electrical Insulation and Dielectric Phenomena, 1995. Annual Report., Conference on, pp. 672-675,1995.

[3] M. Meunier, N. Quirke, and A. Aslanides, "Molecular modeling of electron traps in polymer insulators: Chemical defects and impurities," The Journal of Chemical Physics, vol. 115, no. 6, p. 2876, 2001.

[4] T. Zhou, G. Chen, R. Liao, and Z. Xu, "Charge trapping and detrapping in polymeric materials: Trapping parameters," Journal of Applied Physics, vol. 110, no. 4, 043724, 2011.

[5] N. Liu, and G. Chen, "Modeling of charge trapping/detrapping characteristics in polymer materials and its relation with ageing", Electrical Insulation and Dielectric Phenomena, Annual Report., Conference on, Shenzhen, 2013.

[6] L. Dissado and J. Fothergill, "Electrical degradation and breakdown in polymers", $9^{\text {th }}$ ed., P. N. Morgan, D.V. and K. Overshott, Eds. Peters Peregrinus Ltd., London, United Kingdom, 1992.

[7] L. Dissado, V. Griseri, W. Peasgood, E.S. Cooper, K. Fukunaga, and J. C. Fothergill, "Decay of space charge in a glassy epoxy resin following voltage removal", IEEE Transactions on Dielectrics and Electrical Insulation, vol. 13, no. 4, pp. 903-916, 2006.

[8] V. Afanas, A. Revesz, G. Brown, and H. Hughes, "Deep and Shallow Electron Trapping in the Buried Oxide Layer of SIMOX Structures", Journal of the electrochemical society, vol. 141, no. 10, pp. 2801-2804, 1994.

[9] G. Blaise and W. Sarjeant, "Space charge in dielectrics. Energy storage and transfer dynamics from atomistic to macroscopic scale", IEEE Transactions on Dielectrics and Electrical Insulation, vol. 5, no. 5, pp. 779-808, 1998

[10] J. Simmons and M. Tam, "Theory of isothermal currents and the direct determination of trap parameters in semiconductors and insulators containing arbitrary trap distributions", vol. 7, no. 8, pp. 3706-3713, 1973.

[11] D. Buchanan, M. Fischetti and D. DiMaria, "Coulombic and neutral trapping centers in silicon dioxide." Physical Review B, vol. 43, no. 2, pp. 1471-1486. 